\title{
Feline calicivirus
}

\author{
Alan D. RADFORD*, Karen P. Coyne, Susan Dawson, Carol J. PORTER, \\ Rosalind M. GASKELL
}

\begin{abstract}
University of Liverpool Veterinary Teaching Hospital, Leahurst, Chester High Road, Neston, S. Wirral, CH64 7TE, United Kingdom
\end{abstract}

(Received 23 June 2006; accepted 25 September 2006)

\begin{abstract}
Feline calicivirus (FCV) is an important and highly prevalent pathogen of cats. It belongs to the family Caliciviridae which includes other significant pathogens of man and animals. As an RNA virus, high polymerase error rates convey upon FCV a high genome plasticity, and allow the virus to respond rapidly to environmental selection pressures. This makes the virus very adaptable and has important implications for clinical disease and its control. Being genetically diverse, FCV is associated with a range of clinical syndromes from inapparent infections to relatively mild oral and upper respiratory tract disease with or without acute lameness. More recently, highly virulent forms of the virus have emerged associated with a systemic infection that is frequently fatal. A proportion of FCV infected cats that recover from acute disease, remain persistently infected. In such cats, virus evolution is believed to help the virus to evade the host immune response. Such longterm carriers may only represent a minority of the feline population but are likely to be crucial to the epidemiology of the virus. Vaccination against FCV has been available for many years and has effectively reduced the incidence of clinical disease. However, the vaccines do not prevent infection and vaccinated cats can still become persistently infected. In addition, FCV strain variability means that not all strains are protected against equally. Much progress has been made in understanding the biology and pathogenesis of this important feline virus. Challenges for the future will necessarily focus on how to control the variability of this virus particularly in relation to emerging virulent strains and vaccination.
\end{abstract}

calicivirus / evolution / feline / vaccination / virulence

\section{Table of contents}

1. Aetiology.

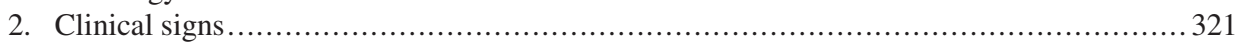

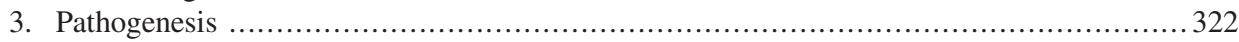

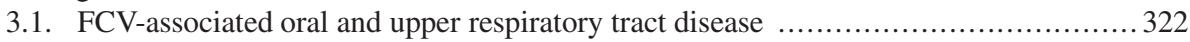

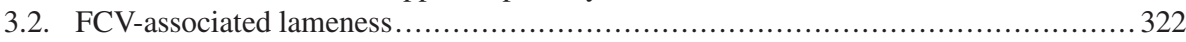

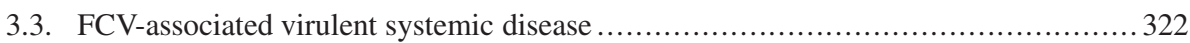

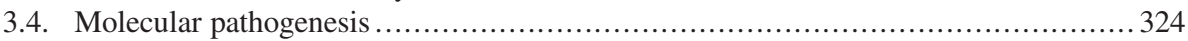

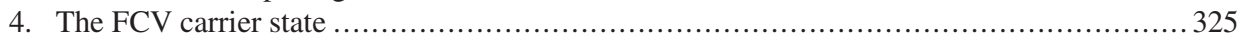

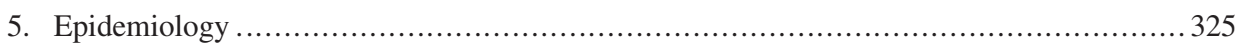

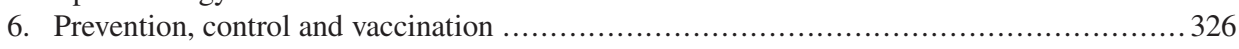

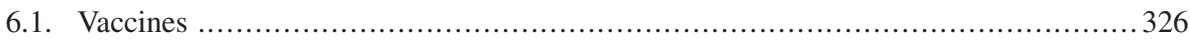

* Corresponding author: alanrad@liv.ac.uk 
6.2. Treatment.

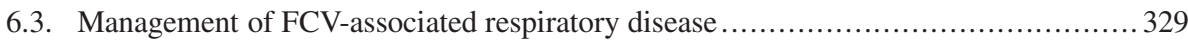

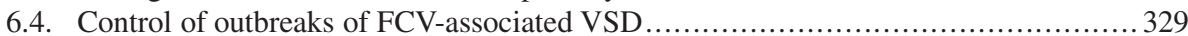

7. Conclusion.....

\section{AETIOLOGY}

Feline calicivirus (FCV) is a highly infectious pathogen of cats with a widespread distribution in the feline population. The virus typically causes moderate, self-limiting acute oral and upper respiratory tract disease. However, some strains induce lameness and recently, more virulent strains have evolved, particularly in the USA.

The virus belongs to the Caliciviridae, a family of viruses which includes important pathogens of man (noroviruses and sapoviruses; together the commonest causes of infectious gastroenteritis in people) and animals including the vesiviruses (e.g. FCV - one of the most consistent clinical signs of FCV is oral vesicles/ulceration) and the lagoviruses (rabbit haemorrhagic disease virus and European brown hare syndrome virus) [35]. Feline calicivirus has a small single-stranded, positive-sense RNA genome of approximately $7.7 \mathrm{~kb}$. The genome is polyadenylated at the 3 ' end, bound by a virally-encoded protein at the 5'-end, and encodes three open reading frames (ORFs). ORF 1 codes for the non-structural proteins including a viral protease and the RNA-dependent RNA polymerase. This polyprotein is posttranslationally cleaved by the viral protease. ORF 2 codes for the major capsid protein which has been divided in to six regions A-F based largely on sequence conservation [99]. Region B, D and F are relatively conserved between FCV isolates, whilst regions $\mathrm{C}$ and $\mathrm{E}$ are variable. Variable region $\mathrm{E}$ is known to contain the major B-cell epitopes $[32,86,110]$ and its variability has been used as the basis of sequence-based methods to differentiate between strains $[84,105]$. Region A is cleaved to produce the mature capsid protein $[10,102]$. ORF 3 encodes for a minor structural protein [103].

Having an RNA genome, we should expect FCV to have a high degree of genomic plasticity. This is a reflection of the lack of proofreading and associated low fidelity generally attributable to viral RNA-dependent RNA polymerases [22]. This mechanism for error-prone replication should afford FCV great adaptability and allow it to exploit new environmental niches. Specific current problems associated with FCV that are a reflection of this include the difficulty of choosing representative strains for inclusion in vaccines, the presence of persistently infected cats, and the emergence of hypervirulent strains of FCV.

The adaptability of FCV is reflected at both genetic and antigenic levels. Phylogenetic analysis of nucleotide sequences mostly from the capsid gene results in a "star-like" phylogeny with little statistical support for sub-species clusters [31,33]. The only exception to this appears to be in viruses from Japan where there is some evidence for the presence of two genotypes [94], although the significance of this is not clear. As a result, it has not proved possible to identify groupings of FCV either on spatial (except possibly Japan), temporal or clinical grounds, and the virus is generally considered to exist as multiple strains that are all members of a single diverse genotype. The definition of "strain" is perhaps somewhat arbitrary, but has been defined by some as FCV isolates that are 
$>20 \%$ different based on nucleotide sequence of capsid region $\mathrm{E}[84,87,88]$.

Observed genetic variability correlates with earlier serological studies. Most FCV isolates can be distinguished antigenically. However, there appears to be sufficient antigenic overlap between isolates to define the viruses as belonging to a single diverse serotype [81,82].

Antigenic and genetic variability is also a feature of other caliciviruses, especially the human noroviruses, where highly variable genogroups containing multiple genotypes are described $[2,34]$. The origins of this diversity are poorly understood. As a result, studies of the evolution of FCV may shed new light on the diversification of other caliciviruses.

\section{CLINICAL SIGNS}

Due to the large number of different strains of $\mathrm{FCV}$, a range of clinical signs may be seen. The most characteristic lesion is oral ulceration, which may often go unreported. Ocular and nasal discharge also frequently occur [8]. Occasionally, inapparent infections or pneumonia may also be seen. Rarely, and usually in young kittens, the more severe respiratory infections can be fatal $[49,60]$. Calicivirus strains can also cause an acute febrile lameness syndrome which has been recreated experimentally $[20,75]$. It has been suggested that lameness and oral/respiratory disease represent two extremes of a clinical continuum, with some individual strains tending to either extreme, and the majority of strains being able to induce both of these clinical signs [108].

More recently, and more worryingly, highly virulent strains of FCV have emerged that are associated with outbreaks of disease with high mortality and a new range of clinical features (FCV-associated virulent systemic disease (VSD) - previously haemorrhagic-like fever) $[42,43,77$,
95]. This is reminiscent of rabbit haemorrhagic disease virus (RHDV). Retrospective studies have shown that RHDV existed in rabbit populations for many years in a seemingly non-pathogenic form $[9,11,70]$. Then, in the 1980s, highly lethal forms were first reported in China, and have since appeared worldwide [58, 72].

As well as upper respiratory tract disease, cats affected by FCV-associated VSD show to varying degrees pyrexia, cutaneous oedema, ulcerative dermatitis, anorexia and jaundice, with up to $50 \%$ of cats dying or being euthanased in extremis. Adult cats are frequently affected more severely than kittens, and worryingly, field vaccination does not appear to be protective. Outbreaks start quickly, generally effect less than 100 animals and disappear rapidly. FCV can be isolated from oral or conjunctival swabs of affected cats. So far, FCV-associated VSD has mainly been reported in the USA. In the UK, one outbreak in 2003 has been described affecting a group of five cats in two households [15]. In addition, the authors are aware of two outbreaks in France ${ }^{1}$ and it is possible that considerably more outbreaks occur than have been reported. VSD has been reproduced experimentally, strongly supporting a role for FCV in this disease [77].

Feline calicivirus has also been associated with other clinical syndromes although these have not been recreated experimentally. The most striking association is with the severe chronic oral disease, lymphoplasmacytic gingivitis stomatitis (LPGS) complex. In some studies, approximately $80 \%$ of cats with LPGS have been shedding FCV compared to $20 \%$ of controls [52, 59, 109]. Although acute faucitis has been reproduced experimentally [91], the chronic disease has not been induced in experimental cats $[53,79]$. It is therefore likely that factors not associated with FCV, including other pathogens [52,

\footnotetext{
${ }^{1}$ Poulet H., personal communication.
} 
59, 116] and host factors [37], may also play a role in this complex and serious syndrome. There have been several attempts to identify consistent genetic and antigenic differences between FCV isolates from cats with LPGS and those from cats with other FCV-associated diseases, and these have met with variable results. Those based on sequencing and monoclonal antibodies have failed to identify consistent differences [31,33,65]. However, those based on polyclonal antisera reactivity have shown some differences between those isolates associated with chronic stomatitis and those associated with other diseases, which has been attributed to evolution of these FCV isolates in such chronically infected cats $[19,79]$.

There has also been some debate about the role of FCV in feline urinary tract disease $[27,92]$. Although virus can be visualized in, and isolated from, urine, there are currently no studies demonstrating a clear association between infection and disease.

\section{PATHOGENESIS}

Cats can be infected with FCV via the nasal, oral or conjunctival routes. The virus replicates mainly in the oral and respiratory tissues, although some strains vary in their tissue tropisms and pathogenicity, such that virus has also been found in visceral tissues, faeces and occasionally in urine. The significance of this to transmission is unknown but is thought to be minimal.

\subsection{FCV-associated oral and upper respiratory tract disease}

Oral ulceration is the most consistent pathological feature of FCV-induced oral and upper respiratory tract disease. Ulcers begin as vesicles, typically on the margin of the tongue but also in other locations. These subsequently rupture, with necrosis of the overlying epithelium and infiltration of neutrophils at the periphery and base [30]. Healing generally takes place over a period of two to three weeks. Pulmonary lesions occur more rarely and appear to result from an initial focal alveolitis, leading to areas of acute exudative pneumonia and then to the development of a proliferative, interstitial pneumonia. Although primary interstitial pneumonia may occur with FCV, it is possible that its importance in natural cases of disease has been over emphasised in the past. This is because many early experimental studies used aerosol challenge to infect cats, rather than the more natural oronasal route of infection.

\subsection{FCV-associated lameness}

Lesions seen in joints of cats with FCVassociated lameness consist of an acute synovitis with thickening of the synovial membrane and an increase in quantity of synovial fluid within the joint [20]. Viral antigen has been identified in macrophagelike cells in the synovial membrane of joints from affected cats [20].

\subsection{FCV-associated virulent systemic disease}

How the pathogenesis of virulent systemic disease (VSD) differs from more typical disease is unknown. However, it is clear that in cases of VSD, virus gains access to cellular compartments not normally associated with FCV. Lesions are widespread and include subcutaneous oedema, ulceration of the mouth, and variable levels of ulceration of the skin particularly on the pinnae and pawpads and nares [77]. Other lesions are more variable and include bronchointerstitial pneumonia and necrosis in the liver, spleen and pancreas. In the most detailed study, viral 
antigen has been detected in the skin, nasal mucosa, lung, pancreas and endothelial cells of the dermis associated with necrosis [78]. Virus particles were also identified by electron microscopy in the cytoplasm and nuclei of lytic epithelial cells undergoing vacuolar degeneration in these lesions. In this study, viral antigen was not detected in the liver of cats despite the presence of pathological lesions. This is in contrast to one study in the UK, in which viral antigen was found in the liver of jaundiced cats [15]. This significance of this discrepancy remains unclear.

It is interesting to speculate on the mechanism of FCV-associated VSD in cats. It is clear that the virus alone is sufficient to cause the disease as the disease has now been re-created experimentally at least twice to the authors' knowledge. This suggests that mutations within the viral genome may be responsible for the highly virulent phenotype. So far, the FCV strains from each reported outbreak of VSD have been genetically distinct from each other. Therefore, if viral mutations are required to cause the hyper-virulent phenotype, then they must evolve independently in each outbreak. Attempts are now being made to identify mutations that are markers of the virulent phenotype. To date, no consistent genetic motif has been reported within the available capsid sequences to differentiate FCV isolates associated with VSD from those associated with more typical FCVassociated disease. However, interestingly, for the two VSD isolates so far sequenced, there is some suggestion that sequence differences lead to the acquisition of an extra glycosylation site in both cases $[1,28]$. This difficulty of identifying clear genomic markers for VSD is not without precedent for FCV, in that attempts to identify markers associated with lameness have also met with failure $[31,33]$. It therefore seems likely that any viral mutations associated with VSD are either subtle, or located in a different region of the genome to that se- quenced to date, or indeed a combination of the two. In addition, it may be possible to evolve virulence by different mutation pathways. This may explain slight differences in the clinical signs and pathology observed in the individual outbreaks.

Most outbreaks of FCV-associated VSD have been associated with the introduction of cats from large rescue colonies into another population [43]. It is possible that the high levels of replication of normal FCV strains in large groups of cats such as rescue shelters may provide the required conditions necessary for the independent emergence of these hypervirulent strains. This is consistent with theories for the evolution of increased virulence in host populations with high levels of non-neutralising immunity [29,61]. Under these conditions, viral variants that are capable of replicating faster and to higher titres will be more likely to be transmitted and therefore positively selected for. Within the originating colony the matched immune response to the virus may damp down these high levels of virus replication in individual cats and therefore the more virulent forms of disease are not seen. However, when these virus strains gain access to naïve populations that have not been exposed to that particular strain before, extreme levels of virus replication lead to the clinical manifestation that is VSD. We have recently shown that virus replication in endemically infected colonies of cats is associated with markedly higher levels of biodiversity than those normally seen within a single strain of FCV [14, 90]. This diversity appears to be driven by immune-mediated positive selection both within individual cats (see carriers below) and associated with transmission between cats. In addition, the high prevalence of FCV in such colonies provides an ideal environment for mixed infections. As a result recombination events between strains, similar to those reported for other caliciviruses [7, 46, 50, 73], have been identified in such colonies, 
and provide a further mechanism for the diversification of viruses [16]. Whether these evolutionary events are associated with the selection of more virulent, faster replicating viral variants remains to be determined.

As stated previously, each outbreak for which sequence data is available has been caused by a distinct strain of FCV. Successive outbreaks are not started by a single virulent strain of FCV that is being widely transmitted. The reasons why each outbreak seems to "burn out" as quickly as it started are unknown but may include, behavioural mechanisms (dying cats are less likely to transmit virus), disease control measures instigated during each outbreak, and evolved attenuation. These virulent FCV outbreaks represent a clear opportunity to explore the mechanisms that underlie both the evolution of virulence and mechanisms of attenuation. One obvious concern is that the virus will evolve to be efficiently transmitted among the cat population. How likely this is to occur is unknown, but if it does, the consequences would be severe, particularly as current vaccines seem to offer little protection.

As well as viral mutations, it is also possible that host and immune factors play a role in this disease. It is certainly true that not all cats die in each outbreak. Some have suggested an immunemediated contribution to the pathogenesis for FCV-associated VSD [15, 28], partly based on the fact that adult cats seem to develop more severe disease than young animals [43]. A possible immune-mediated pathogenesis has been shown for other FCV infections such as lameness [4], and in some cases, vaccination appears to potentiate FCV infection [17].

Although outbreaks of VSD have only recently been described it is interesting to note that several case reports in the past have described similar clinical findings in FCV infected cats including jaundice [26] and sudden death [60].

\subsection{Molecular pathogenesis}

The study of the molecular mechanism by which FCV induces disease has benefited considerably from the fact that FCV grows well in cell culture, and because an infectious clone is available [101]. This is in contrast to other members of the Caliciviridae such as human noro- and sapoviruses which do not grow in cell culture, and where infectious clones are not available. This has led to the use of FCV infection as a model of calicivirus molecular biology.

In cell culture, infected cells show a characteristic cytopathic effect associated with cell rounding and membrane blebbing [51]. Under these conditions, infection with FCV leads to an inhibition of cellular protein synthesis (shut-off) associated with cleavage of the host translation initiation factors [117]. Such a mechanism may allow the virus to divert the cellular translation machinery from cap dependant to cap-independent translation thereby stopping translation of cellular mRNAs and allowing translation to focus on the viral VpG-bound RNA. A similar system is also used by the closely related picornaviruses.

Molecular studies have now shown that virus infection in cell culture triggers the mitochondrial pathway, leading to caspase activation and apoptosis [71,104]. Whether this is what happens in the entire animal remains unknown.

A big break through for FCV research was recently reported when the junctional adhesion molecule-1 (JAM-1) was identified as a cellular receptor for FCV in cell culture [62]. Transfection of nonpermissive cells with a JAM-1 expression system rendered the cell line permissive for FCV and anti-feline JAM-1 antibodies reduced replication of $\mathrm{FCV}$ in permissive cells. Whether strains of FCV associated with different types of disease use different receptors remains to be determined. In human noroviruses, different host expression 
of viral receptors belonging to the $\mathrm{ABH}$ histo-blood group family is associated with resistant to infection in some individuals $[38,44,57]$. Whether a similar mechanism operates for FCV is unknown although interestingly in the field, some cats do appear to be relatively resistant to infection despite between heavily exposed to challenge virus [14].

\section{THE FCV CARRIER STATE}

Most cats shed FCV in oropharyngeal secretions for 30 days after infection. Carriers are somewhat arbitrarily defined as those cats that shed virus beyond this period. Some individual FCV carriers may shed virus for life, although most cats appear to spontaneously eliminate the virus. In one experimental study, the duration of FCV shedding by carrier cats was shown to broadly conform to a half-life such that every 75 days, half of a group of positive cats cease to shed the virus [115]. Although an oversimplification, with many factors likely to affect the duration of shedding, this half-life model of the FCV carrier state remains a useful one.

Studies of endemically infected colonies seem to confirm these experimental observations, that long-term persistence is indeed a relatively rare event [14]. In such colonies, only a minority of cats that are continuously shedding FCV are actually infected with the same lineage of virus over the course of this infection. We refer to these cats as progressive evolvers. These cats are likely to provide a reservoir of virus to colonies, allowing the virus to persist in what is otherwise a very small population. By contrast, the majority of cats that shed virus over prolonged periods of time seem to do so by being reinfected either with diverse viral variants of the same strain or with distinct strains that are co-circulating within the household [14].

Progressive evolver carrier cats shed virus more-or-less continuously and so are likely to represent a constant source of infection to susceptible cats. In addition, some carrier cats appear to shed higher levels of virus than others, and these cats are likely to be of greater epidemiological significance [14, 114].

The mechanism of persistence for FCV is not fully elucidated. Virus has been found in tonsils of carrier cats, suggesting the virus may replicate within immuneprivileged sites. However, tonsilectomy does not eliminate the carrier state, and therefore it is clear that the virus persists in other as yet undefined extra-tonsillar sites. Several studies have looked at virus evolution in progressively-evolving carrier cats and suggested that, as for other persistent RNA viruses, viral evolution, particularly in immunodominant regions of the capsid protein, lead to antigenic variation, and allow the virus to evade the developing host immune response $[48,54,85]$. It is likely that a combination of both viral and host factors will ultimately determine the outcome and duration of infection.

\section{EPIDEMIOLOGY}

Feline calicivirus infection is widespread in the general cat population [5, $36,68,107]$. The prevalence is generally broadly proportional to the number of cats in the household, with the highest prevalence usually seen where large groups of cats are housed together. As a result, privately owned pet cats kept in small numbers generally have relatively low prevalence $(\sim 10 \%)$ [113]. In contrast, random cats living in colonies or shelters usually have a higher chance of being infected $(\sim 25 \%-40 \%)$ [3, 13, 40, 88, 113]. The prevalence within individual colonies is very variable. In some circumstances the prevalence appears to be low $[14,41,89]$, whereas in other colonies we have found prevalence values of $50-90 \%$ over prolonged periods of time $[14,90]$. 
Within the cat population, FCV is present in acutely infected cats and in clinically-recovered carrier cats. The virus can also persist in the environment for several days to several weeks on dried surfaces at room temperature, and longer in colder wetter conditions [12, 23, 24]. Indirect transmission can therefore occur, especially within the close confines of a cattery where secretions may contaminate cages, feeding and cleaning utensils or personnel. It is generally accepted that there are no known reservoirs or alternative hosts for FCV, and in utero transmission does not seem to occur. However interestingly, as well as having there own specific canine calicivirus, FCV-like viruses have also been isolated from dogs $[39,64,93]$. The role of these viruses in the epidemiology of FCV in the cat (and dog) is uncertain. In one study, an association was shown between the presence of dogs and FCV infection in cats [5], whilst a second study has suggested cat households with dogs have a lower prevalence of FCV infection [40].

The immune response has a somewhat limited impact on FCV infection. It is clear that pre-existing immunity, acquired either naturally as maternal-derived antibodies (MDA) or artificially following vaccination, can reduce or eliminate the clinical signs of subsequent FCV challenge. However, such pre-existing immunity does not prevent infection and these animals may become carriers following sub-clinical infection with field virus. As with other significant pathogens, these "silent-carriers" are likely to play a crucial role in the epidemiology of this disease. There is no evidence that vaccination will 'cure' an existing carrier state.

\section{PREVENTION, CONTROL AND VACCINATION}

The main-stay of FCV-associated disease control in the cat population is vac- cination. In pet cats living in small populations, this is likely to be sufficient. In larger groups of cats, where the prevalence and amount of virus shed is likely to be higher, vaccination needs to be accompanied by careful management procedures. Treatment is non-specific. The control of outbreaks of FCV-associated VSD will be considered separately.

\subsection{Vaccines}

Several types of vaccines are now available for FCV. They are generally considered to be safe and effective at reducing or preventing classical oral/respiratory disease, but do not protect against infection or the development of the carrier state. Evidence from the field suggests that the current vaccines do not prevent FCVassociated VSD with outbreaks occurring in vaccinated cats $[15,42,77,95]$. However, there is some experimental data to support their use $[6,77]$. The impact of vaccination on LPGS is unclear. Vaccinated cats certainly develop LPGS and no vaccine carries a data sheet claim for preventing this disease. This is consistent with the uncertain role of FCV in this syndrome.

All licensed FCV vaccines are based on whole viral antigens grown in cell culture. Most are monovalent (based on a single strain), although recently a bivalent vaccine has been licensed [80]. Live-attenuated and inactivated (both adjuvanted and non-adjuvanted) vaccines are available in most countries, and are given parenterally. However, in some parts of the world, live-attenuated vaccines are licensed for intranasal use.

Live intranasal vaccines induce local mucosal immunity, and this is probably more effective than immunity induced by parenteral vaccines. However, because the virus replicates at the site of inoculation, clinical signs such as mild sneezing may be seen after several days in some individuals $[55,74]$. Where available, intranasal 
vaccines are particularly useful when a rapid onset of protection is required e.g. for a cat going into a boarding cattery or in the face of an outbreak of disease. In contrast to parenteral vaccines, only a single dose of intranasal vaccine is generally required to induce immunity following primary vaccination. Most published studies have used a feline herpesvirus challenge where high levels of protection have been shown four days after intranasal vaccination and partial protection after two $[55,74]$. These vaccines may also overcome MDA better than parenteral vaccines, although in general their use is only licensed in kittens from 12 weeks of age. Live intranasal vaccines have shown an increase in popularity amongst some veterinarians, in part due to public concerns about the role of inactivated vaccines in injection site reactions and sarcomas.

Recommended vaccination schedules tend to be of the "traditional" type with a primary course at 8-9 and 12 weeks followed by annual boosters. Some are licensed for earlier use [21]. There is now evidence to suggest that not all kittens are able to respond to vaccination at 12 weeks of age, such that under some circumstances, later kitten vaccinations may be needed to overcome persistent MDA [21, 47].

As with other small animal vaccines, the requirement for annual booster vaccinations is currently being debated. It will be important to consider the antigenic diversity of FCV as this debate is developed. Moderate levels of virus neutralizing antibody have been shown to persist in a group of vaccinated cats for at least four years, although after 7.5 years, titers had declined to low or non-detectable levels [97, 98]. Protection against FCV challenge decreased from $85 \%$ three weeks after vaccination to $63 \%$ after 7.5 years. However, this study represented a homologous challenge, with both the vaccine and the challenge virus being based on strain 255, which is the USDA-approved FCV challenge in the USA. It is therefore likely that shorter durations of immunity/protection would have been seen with a heterologous challenge, although how much shorter is unknown.

As with most vaccines, there are well documented adverse reactions in a minority of cases. Along with other injections, adjuvanted vaccines have been associated with injection-site reactions and sarcomas [69]. Parenteral modified-live FCV vaccines have been associated with clinical signs in the immediate period postvaccination. Most of these appear to be due to coincidental infection with field virus although in some cases, sequence analysis has shown that vaccine virus may be involved [84, 87]. Vaccine virus has also occasionally been detected circulating in the cat population, though the significance of this is not yet known $[77,88,89]$. A third potential problem for current vaccines is the antigenic variability of FCV strains, which means that no vaccine is likely to be able to neutralise all field isolates of virus such that occasional vaccine breakdowns can occur [18]. In order to try and circumvent some of these problems, a bivalent inactivated, non-adjuvanted vaccine has recently been marketed in Europe [80]. The success of this in controlling disease and in the market place remains to be determined.

The areas where vaccines could be improved seem clear, and pose a considerable challenge. Firstly, it will be important to increase the cross-reactivity of vaccines to maximise the chances of cross-protection, minimise the number of vaccine breakdowns, and minimise the possibilities of evolving vaccine resistant strains [56]. This approach will hopefully help with the control of both typical and more virulent forms of disease. The success of specifically including antigens from outbreaks of VSD in vaccines to protect against these more severe forms of disease is uncertain 
since to date, each strain of FCV associated with VSD appears to be different [15].

A second approach to improving existing vaccines is to develop immune responses that reduce or eliminate challenge virus shedding and prevent or reduce the likelihood of vaccinated cats becoming persistently infected. It is certainly true that existing vaccines do not prevent infection. Indeed there is a small amount of experimental data to suggest that under some circumstances, challenge virus may be shed for longer and at a higher titre by cats with some previous immunity when compared to naïve cats [17]. Whether this can also occur with commercial vaccines is not known. Whilst one vaccine licensed in the EU has a claim to reduce FCV shedding, the effect of most vaccines on viral shedding is generally not publicly available. Reducing or eliminating challenge virus shedding in vaccinated cats is likely to be a "holy-grail" of vaccination, not just for FCV but for many other important pathogens. Whilst this is an immense challenge, we will only begin to address it when the monitoring of FCV shedding patterns in vaccinated and challenged cats becomes more routine.

The mechanisms by which cats are protected against clinical disease are still unclear. Antibody is considered to be most important for protection, and is routinely measured in cell culture by virus neutralisation tests. Such tests have been used to identify those strains that induce the most broadly cross-reactive immune response for use as vaccine antigens [19, $76,80]$. Available cross-protection studies suggest that the correlation between titre and protection is reasonable [81]. However, in challenge studies, some cats with no measurable antibody were also protected against clinical disease [53]. This suggests that cell mediated and innate immune responses also play an important part in protection. Lack of clear biomarkers for protection represent a bottleneck to vaccine development, especially as we seek to rationally improve the repertoire of field strains covered by individual vaccines. Therefore, in the short-medium term, challenge experiments will remain a gold-standard for measuring FCV vaccine efficacy. However, the antigenic variability of FCV complicates both the rationale choice of challenge virus, and the interpretation of challenge experiments based on single strains. The most sensible approach will be to test vaccines against a panel of representative FCV challenge isolates. However, this rigorous experimental approach will be rendered impractical by the commercial and welfare implications of what would become very large experiments. Perhaps in the future, the ultimate testing of the efficacy of FCV vaccination may need to take place in rigorous phased clinical trials as occurs in human medicine.

There are some published reports of novel vaccine technologies including DNA vaccination [100], myxoma recombinants [66, 67], and herpesvirus recombinants [118] for FCV. Such vaccines could offer several potential advantages over those based on conventional technologies including inability to induce FCV infection, and induction of a local mucosal immune response. However, to date, none are available commercially.

\subsection{Treatment}

Broad-spectrum antibiotic treatment is generally recommended in cases of more severe FCV-associated oral and respiratory disease to minimize potential complications associated with secondary bacterial infection. As swallowing may be painful, antibiotics can be given either as syrups (if available) or parenterally. Good nursing care, with regular cleansing of discharges, is essential. The cat should be encouraged to eat by offering strongly-flavoured aromatic foods. If eating is painful, liquidized or specialized proprietary foods may be 
of some help. In some cases, the use of appetite stimulants such as diazepam or cyproheptadine may also be of some benefit. Some severely affected cases may require fluid therapy, and where anorexia is prolonged, an oesophagostomy or gastrotomy tube may be indicated.

For some viral diseases of humans, both specific and more broad-spectrum therapies have made it to clinical practice. Specific antivirals for veterinary pathogens are unlikely to be developed in the near future due to the prohibitive costs. Although some broad-spectrum antivirals are effective against FCV in cell culture, they are too toxic for use in the cat [83]. Interferon is used by some, although to the authors' knowledge, published evidence for its effectiveness remains limited to in vitro studies [106], and it is not licensed for control of FCV-disease in Europe. Experimental trials of chimeric mouse-cat monoclonal antibodies have shown some promise in treatment $[111,112]$.

\subsection{Management of FCV-associated respiratory disease}

In practice, controlling FCV is usually associated with a similar need to control feline herpesvirus. A more detailed description of the control measures for these two pathogens is given elsewhere [30]. Briefly, the control measures for FCVassociated respiratory disease depend entirely on the population of cats. For individual household cats where prevalence is low, vaccination is likely to be sufficient. In boarding and rescue catteries, vaccination, quarantine facilities, batching of arrivals, good husbandry/hygiene, well designed pens which prevent direct contact between cats, and avoiding overcrowding are critical to minimising viral loads and the spread of virus through the population. Molecular studies on FCV suggest that if this is done well, FCV transmission can be minimal [88]. In breeding colonies that are known to be free of virus (e.g. research colonies), inactivated vaccines may be used and animals coming into the colony (stud cats, replacements) should ideally be sourced from similar FCV-negative colonies, and should be quarantined and tested on several occasions to determine if they are free of the virus. In endemically infected breeding colonies control is generally aimed at reducing clinical disease. Specific measures include reducing stocking density, early weaning kittens into isolation, and early vaccination of kittens. Vaccinating pregnant queens may reduce disease in young kittens by boosting their MDA [45]. However, the safety of vaccines in pregnant queens is largely unknown and clinicians should consult data sheets. Live vaccines should be avoided, particularly those containing live feline parvovirus. It may be possible to eradicate virus from such colonies by a test and remove strategy. However, in practice, this is likely to be extremely difficult.

\subsection{Control of outbreaks of FCV-associated VSD}

Diagnosing FCV-associated VSD in the cat remains somewhat of a conundrum since there are no unique clinical or laboratory markers for the disease. Although the clinical features can be quite striking with relatively few differential diagnoses, FCV strain variability means that each outbreak can be associated with slightly different clinical signs. The authors are frequently asked about individual cats with suspicious signs, some of which are also FCV positive. Such individual cats must be treated carefully, but whether these represent actual sporadic cases of VSD is unknown. The index of suspicion for VSD increases dramatically when two or more cats present with the same clinical signs. However, ultimately as we seek to understand the mechanism of this disease, 
experimental infections with viruses isolated from such cats will be required to categorically identify true outbreaks of FCVassociated VSD.

Crucial to the control of suspect outbreaks of this severe disease is early recognition/suspicion and prompt rigorous biosecurity. Early in the outbreak, it is very important that owners and staff are all aware of the disease and what it looks like. This includes veterinary surgeons at neighbouring practices who may see other cases from the same outbreak. Specific measures must include contact tracing and quarantining of all suspect cats. Actual and suspect cases are perhaps best managed away from the veterinary hospital. However, where cases must be hospitalised, they must be kept in strict isolation and barrier nursed. All staff should be made aware of the case, and those who do not own cats should preferably be involved in the treatment of the affected animal.

The virus is generally considered to survive for up to two weeks in the environment and therefore can be transmitted by direct contact and fomites, and possibly by aerosol. Affected cats are likely to shed virus from their respiratory and oral secretions, but any discharge from skin may also be highly infectious. FCV has also been isolated from faeces and urine. Areas where the cat has been and any equipment require disinfection using a product that has been shown to be effective against FCV. Quaternary ammonium compounds are generally not thought to be completely effective $[23,25,42,63]$. However, a 1:32 dilution of household bleach with some household detergent does seem to be effective providing contact time is sufficient [96]. With these measures, all outbreaks seem to have been successfully managed. This is supported by the molecular epidemiology which so far has suggested that each outbreak is associated with a distinct strain of virus. Whether the end of each outbreak is entirely attributable to disease control measures or includes a cat behavioural and/or virus evolutionary component, remains to be determined.

\section{CONCLUSION}

Feline calicivirus has been recognised as an important pathogen of cats for over 40 years now. As an RNA pathogen, it seems to rely on evolution to maintain itself in the population. Where the virus came from we do not know. Today we struggle with newly emerged virulent strains and have a clear need to improve the cross-protection offered by our vaccines. What is certainly true is that FCV is still changing. We must expect the clinical features of this virus to change and accordingly, our attempts to control it will need to be continually updated. Because of its high prevalence, FCV also represents a good model system in which to explore the consequences of virus evolution in its natural host. Sounds interesting.

\section{REFERENCES}

[1] Abd-Eldaim M., Potgieter L., Kennedy M., Genetic analysis of feline caliciviruses associated with a hemorrhagic-like disease, J. Vet. Diagn. Invest. (2005) 17:420-429.

[2] Ando T., Noel J.S., Fankhauser R.L., Genetic classification of "Norwalk-like viruses", J. Infect. Dis. (2000) 181:S336348 .

[3] Bannasch M.J., Foley J.E., Epidemiologic evaluation of multiple respiratory pathogens in cats in animal shelters, J. Feline Med. Surg. (2005) 7:109-119.

[4] Bennett D., Gaskell R.M., Mills A., Knowles J., Carter S., McArdle F., Detection of feline calicivirus antigens in the joints of infected cats, Vet. Rec. (1989) 124:329-332.

[5] Binns S.H., Dawson S., Speakman A.J., Cuevas L.E., Hart C.A., Gaskell C.J., Morgan K.L., Gaskell R.M., A study of feline upper respiratory tract disease with reference to prevalence and risk factors for infection with feline calicivirus and feline herpesvirus, J. Feline Med. Surg. (2000) 2:123-133. 
[6] Brunet S., Jas D., David F., Bublot M., Poulet H., Feline calicivirus: vaccinations against virulent strains, in: Comparative and emerging virus infections of dogs and cats, 2005, Liverpool.

[7] Bull R.A., Hansman G.S., Clancy L.E., Tanaka M.M., Rawlinson W.D., White P.A., Norovirus recombination in ORF1/ORF2 overlap, Emerg. Infect. Dis. (2005) 11:1079-1085.

[8] Cai Y., Fukushi H., Koyasu S., Kuroda E., Yamaguchi T., Hirai K., An etiological investigation of domestic cats with conjunctivitis and upper respiratory tract disease in Japan, J. Vet. Med. Sci. (2002) 64:215-219.

[9] Capucci L., Fusi P., Lavazza A., Pacciarini M.L., Rossi C., Detection and preliminary characterization of a new rabbit calicivirus related to rabbit hemorrhagic disease virus but nonpathogenic, J. Virol. (1996) 70:8614-8623.

[10] Carter M.J., Milton I.D., Turner P.C., Meanger J., Bennett M., Gaskell R.M., Identification and sequence determination of the capsid protein gene of feline calicivirus, Arch. Virol. (1992) 122:223-235.

[11] Chasey D., Rabbit haemorrhagic disease: the new scourge of Oryctolagus cuniculus, Lab. Anim. (1997) 31:33-44.

[12] Clay S., Maherchandani S., Malik Y.S., Goyal S.M., Survival on uncommon fomites of feline calicivirus, a surrogate of noroviruses, Am. J. Infect. Control (2006) 34:41-43.

[13] Coutts A.J., Dawson S., Willoughby K., Gaskell R.M., Isolation of feline respiratory viruses from clinically healthy cats at UK cat shows, Vet. Rec. (1994) 135:555-556.

[14] Coyne K.P., Dawson S., Radford A.D., Cripps P.J., Porter C.J., McCracken C.M., Gaskell R.M., Long term analysis of feline calicivirus prevalence and viral shedding patterns in naturally infected colonies of domestic cats, Vet. Microbiol. (2006) 118: 12-25.

[15] Coyne K.P., Jones B.R., Kipar A., Chantrey J., Porter C.J., Barber P.J., Dawson S., Gaskell R.M., Radford A.D., Lethal outbreak of disease associated with feline calicivirus infection in cats, Vet. Rec. (2006) 158:544-550.

[16] Coyne K.P., Reed F.C., Porter C.J., Dawson S., Gaskell R.M., Radford A.D., Recombination of feline calicivirus within an endemically infected cat colony, J. Gen. Virol. (2006) 87:921-926.

[17] Dawson S., Smyth N.R., Bennett M., Gaskell R.M., McCracken C.M., Brown A.,
Gaskell C.J., Effect of primary-stage feline immunodeficiency virus infection on subsequent feline calicivirus vaccination and challenge in cats, AIDS (1991) 5:747-750.

[18] Dawson S., McArdle F., Bennett D., Carter S.D., Bennett M., Ryvar R., Gaskell R.M., Investigation of vaccine reactions and breakdowns after feline calicivirus vaccination, Vet. Rec. (1993) 132:346-350.

[19] Dawson S., McArdle F., Bennett M., Carter M., Milton I.P., Turner P., Meanger J., Gaskell R.M., Typing of feline calicivirus isolates from different clinical groups by virus neutralisation tests, Vet. Rec. (1993) 133:13-17.

[20] Dawson S., Bennett D., Carter S.D., Bennett M., Meanger J., Turner P.C., Carter M.J., Milton I., Gaskell R.M., Acute arthritis of cats associated with feline calicivirus infection, Res. Vet. Sci. (1994) 56:133-143.

[21] Dawson S., Willoughby K., Gaskell R.M., Wood G., Chalmers W.S., A field trial to assess the effect of vaccination against feline herpesvirus, feline calicivirus and feline panleucopenia virus in 6-week-old kittens, J. Feline Med. Surg. (2001) 3:17-22.

[22] Domingo E., Menendez-Arias L., Holland J.J., RNA virus fitness, Rev. Med. Virol. (1997) 7:87-96.

[23] Doultree J.C., Druce J.D., Birch C.J., Bowden D.S., Marshall J.A., Inactivation of feline calicivirus, a Norwalk virus surrogate, J. Hosp. Infect. (1999) 41:51-57.

[24] Duizer E., Bijkerk P., Rockx B., De Groot A., Twisk F., Koopmans M., Inactivation of caliciviruses, Appl. Environ. Microbiol. (2004) 70:4538-4543.

[25] Eleraky N.Z., Potgieter L.N., Kennedy M.A., Virucidal efficacy of four new disinfectants, J. Am. Anim. Hosp. Assoc. (2002) 38:231-234.

[26] Ellis T.M., Jaundice in a siamese cat with in utero feline calicivirus infection, Aust. Vet. J. (1981) 57:383-385.

[27] Fabricant C.G., Rich L.J., Microbial studies of feline urolithiasis, J. Am. Vet. Med. Assoc. (1971) 158:976-980.

[28] Foley J., Hurley K., Pesavento P.A., Poland A., Pedersen N.C., Virulent systemic feline calicivirus infection: local cytokine modulation and contribution of viral mutants, J. Feline Med. Surg. (2006) 8:55-61.

[29] Gandon S., Mackinnon M.J., Nee S., Read A.F., Imperfect vaccines and the evolution of pathogen virulence, Nature (2001) 414:751-756. 
[30] Gaskell R.M., Radford A.D., Dawson S., Feline infectious respiratory disease, in: Chandler E.A., Gaskell C.J., Gaskell R.M. (Eds.), Feline medicine and therapeutics, 2004, Blackwell Publishing, pp. 577-595.

[31] Geissler K., Schneider K., Platzer G., Truyen B., Kaaden O.-R., Truyen U., Genetic and antigenic heterogeneity among feline calicivirus isolates from distinct disease manifestations, Virus Res. (1997) 48:193-206.

[32] Geissler K., Schneider K., Truyen U., Mapping neutralizing and non-neutralizing epitopes on the capsid protein of feline calicivirus, J. Vet. Med. B Infect. Dis. Vet. Public Health (2002) 49:55-60.

[33] Glenn M., Radford A.D., Turner P.C., Carter M., Lowery D., DeSilver D.A., Meanger J., Baulch-Brown C., Bennett M., Gaskell R.M., Nucleotide sequence of UK and Australian isolates of feline calicivirus (FCV) and phylogenetic analysis of FCVs, Vet. Microbiol. (1999) 67:175-193.

[34] Green J., Vinje J., Gallimore C.I., Koopmans M., Hale A., Brown D.W., Clegg J.C., Chamberlain J., Capsid protein diversity among Norwalk-like viruses, Virus Genes (2000) 20:227-236.

[35] Green K.Y., Ando T., Balayan M.S., Berke T., Clarke I.N., Estes M.K., Matson D.O., Nakata S., Neill J.D., Studdert M.J., Thiel H.J., Taxonomy of the caliciviruses, J. Infect. Dis. (2000) 181:S322-S330.

[36] Harbour D.A., Howard P.E., Gaskell R.M., Isolation of feline calicivirus and feline herpesvirus from domestic cats 1980 to 1989 , Vet. Rec. (1991) 128:77-80.

[37] Harley R., Helps C.R., Harbour D.A., Gruffydd-Jones T.J., Day M.J., Cytokine mRNA expression in lesions in cats with chronic gingivostomatitis, Clin. Diagn. Lab. Immunol. (1999) 6:471-478.

[38] Harrington P.R., Vinje J., Moe C.L., Baric R.S., Norovirus capture with histo-blood group antigens reveals novel virus-ligand interactions, J. Virol. (2004) 78:3035-3045.

[39] Hashimoto M., Roerink F., Tohya Y., Mochizuki M., Genetic analysis of the RNA polymerase gene of caliciviruses from dogs and cats, J. Vet. Med. Sci. (1999) 61:603608.

[40] Helps C.R., Lait P., Damhuis A., Bjornehammar U., Bolta D., Brovida C., Chabanne L., Egberink H., Ferrand G., Fontbonne A., Pennisi M.G., GruffyddJones T., Gunn-Moore D., Hartmann K., Lutz H., Malandain E., Mostl K., Stengel C., Harbour D.A., Graat E.A., Factors associated with upper respiratory tract disease caused by feline herpesvirus, feline calicivirus, Chlamydophila felis and Bordetella bronchiseptica in cats: experience from 218 European catteries, Vet. Rec. (2005) 156:669-673.

[41] Holst B.S., Berndtsson L.T., Englund L., Isolation of feline herpesvirus- 1 and feline calicivirus from healthy cats in Swedish breeding catteries, J. Feline Med. Surg. (2005) 7:325-331.

[42] Hurley K.E., Pesavento P.A., Pedersen N.C., Poland A.M., Wilson E., Foley J.E., An outbreak of virulent systemic feline calicivirus disease, J. Am. Vet. Med. Assoc. (2004) 224:241-249.

[43] Hurley K.F., Sykes J.E., Update on feline calicivirus: new trends, Vet. Clin. North Am. Small Anim. Pract. (2003) 33:759_ 772.

[44] Hutson A.M., Atmar R.L., Marcus D.M., Estes M.K., Norwalk virus-like particle hemagglutination by binding to $\mathrm{h}$ histoblood group antigens, J. Virol. (2003) 77:405-415

[45] Iglauer F., Gartner K., Morstedt R., Maternal protection against feline respiratory disease by means of booster vaccinations during pregnancy - a retrospective clinical study, Kleintierpraxis (1989) $34: 235$

[46] Jiang X., Espul C., Zhong W.M., Cuello H., Matson D.O., Characterization of a novel human calicivirus that may be a naturally occurring recombinant, Arch. Virol. (1999) 144:2377-2387.

[47] Johnson R.P., Povey R.C., Transfer and decline of maternal antibody to feline calicivirus, Can. Vet. J. (1983) 24:6-9.

[48] Johnson R.P., Antigenic change in feline calicivirus during persistent infection, Can. J. Vet. Res. (1992) 56:326-330.

[49] Kahn D.E., Gillespie J.H., Feline viruses: Pathogenesis of picornavirus infection in the cat, Am. J. Vet. Res. (1971) 32:521531.

[50] Katayama K., Miyoshi T., Uchino K., Oka T., Tanaka T., Takeda N., Hansman G.S., Novel recombinant sapovirus, Emerg. Infect. Dis. (2004) 10:1874-1876.

[51] Knowles J.O., Studies on feline calicivirus with particular reference to chronic stomatitis in the cat, 1988, University of Liverpool.

[52] Knowles J.O., Gaskell R.M., Gaskell C.J., Harvey C.E., Lutz H., Prevalence of feline calicivirus, feline leukaemia virus and 
antibodies to FIV in cats with chronic stomatitis, Vet. Rec. (1989) 124:336-338.

[53] Knowles J.O., McArdle F., Dawson S., Carter S.D., Gaskell C.J., Gaskell R.M., Studies on the role of feline calicivirus in chronic stomatitis in cats, Vet. Microbiol. (1991) 27:205-219.

[54] Kreutz L.C., Johnson R.P., Seal B.S., Phenotypic and genotypic variation of feline calicivirus during persistent infection of cats, Vet. Microbiol. (1998) 59:229-236.

[55] Lappin M.R., Sebring R.W., Porter M., Radecki S.J., Veir J., Effects of a single dose of an intranasal feline herpesvirus 1, calicivirus, and panleukopenia vaccine on clinical signs and virus shedding after challenge with virulent feline herpesvirus $1, \mathrm{~J}$. Feline Med. Surg. (2006) 8:158-163.

[56] Lauritzen A., Jarrett O., Sabara M., Serological analysis of feline calicivirus isolates from the United States and United Kingdom, Vet. Microbiol. (1997) 56:55-63.

[57] Lindesmith L., Moe C., Marionneau S., Ruvoen N., Jiang X., Lindblad L., Stewart P., LePendu J., Baric R., Human susceptibility and resistance to Norwalk virus infection, Nat. Med. (2003) 9:548-553.

[58] Liu S.J., Xue H.P., Pu B.Q., Qian N.H., A new viral disease in rabbits, Animal Husbandry and Veterinary Medicine (1984) 16:253-255.

[59] Lommer M.J., Verstraete F.J., Concurrent oral shedding of feline calicivirus and feline herpesvirus 1 in cats with chronic gingivostomatitis, Oral Microbiol. Immunol. (2003) 18:131-134.

[60] Love D.N., Baker K.D., Sudden death in kittens associated with a feline picornavirus, Aust. Vet. J. (1972) 48:643.

[61] Mackinnon M.J., Read A.F., Virulence in malaria: an evolutionary viewpoint, Philos. Trans. R Soc. Lond. B Biol. Sci. (2004) 359:965-986.

[62] Makino A., Shimojima M., Miyazawa T., Kato K., Tohya Y., Akashi H., Junctional adhesion molecule 1 is a functional receptor for feline calicivirus, J. Virol. (2006) $80: 4482-4490$

[63] Marks P.J., Vipond I.B., Carlisle D., Deakin D., Fey R.E., Caul E.O., Evidence for airborne transmission of Norwalk-like virus (NLV) in a hotel restaurant, Epidemiol. Infect. (2000) 124:481-487.

[64] Martella V., Pratelli A., Gentile M., Buonavoglia D., Decaro N., Fiorente P., Buonavoglia C., Analysis of the capsid protein gene of a feline-like calicivirus isolated from a dog, Vet. Microbiol. (2002) 85:315322.

[65] McArdle F., Dawson S., Carter M.J., Milton I.D., Turner P.C., Meanger J., Bennett M., Gaskell R.M., Feline calicivirus strain differentiation using monoclonal antibody analysis in an enzyme-linked immunoflow-assay, Vet. Microbiol. (1996) 51:197206.

[66] McCabe V.J., Tarpey I., Spibey N., Vaccination of cats with an attenuated recombinant myxoma virus expressing feline calicivirus capsid protein, Vaccine (2002) 20:2454-2462.

[67] McCabe V.J., Spibey N., Potential for broad-spectrum protection against feline calicivirus using an attenuated myxoma virus expressing a chimeric FCV capsid protein, Vaccine (2005) 23:5380-5388.

[68] Mochizuki M., Kawakami K., Hashimoto M., Ishida T., Recent epidemiological status of feline upper respiratory infections in Japan, J. Vet. Med. Sci. (2000) 62:801-803.

[69] Morrison W.B., Starr R.M., Vaccineassociated feline sarcomas, J. Am. Vet. Med. Assoc. (2001) 218:697-702.

[70] Nagesha H.S., McColl K.A., Collins B.J., Morrissy C.J., Wang L.F., Westbury H.A., The presence of cross-reactive antibodies to rabbit haemorrhagic disease virus in Australian wild rabbits prior to the escape of virus from quarantine, Arch. Virol. (2000) 145:749-757.

[71] Natoni A., Kass G.E., Carter M.J., Roberts L.O., The mitochondrial pathway of apoptosis is triggered during feline calicivirus infection, J. Gen. Virol. (2006) 87:357-361.

[72] Ohlinger V.F., Haas B., Meyers G., Weiland F., Thiel H.J., Identification and characterization of the virus causing rabbit hemorrhagic disease, J. Virol. (1990) 64:33313336.

[73] Oliver S.L., Brown D.W., Green J., Bridger J.C., A chimeric bovine enteric calicivirus: evidence for genomic recombination in genogroup III of the Norovirus genus of the Caliciviridae, Virology (2004) 326:231239.

[74] Orr C.M., Gaskell C.J., Gaskell R.M., Interaction of an intranasal combined feline viral rhinotracheitis, feline calicivirus vaccine and the FVR carrier state, Vet. Rec. (1980) 106:164-166.

[75] Pedersen N.C., Laliberte L., Ekman S., A transient febrile limping syndrome of kittens caused by two different strains of feline calicivirus, Feline Pract. (1983) 13:26-35. 
[76] Pedersen N.C., Hawkins K.F., Mechanisms of persistence of acute and chronic feline calicivirus infections in the face of vaccination, Vet. Microbiol. (1995) 47:141-156.

[77] Pedersen N.C., Elliott J.B., Glasgow A., Poland A., Keel K., An isolated epizootic of hemorrhagic-like fever in cats caused by a novel and highly virulent strain of feline calicivirus, Vet. Microbiol. (2000) 73:281300.

[78] Pesavento P.A., MacLachlan N.J., DillardTelm L., Grant C.K., Hurley K.F., Pathologic, immunohistochemical, and electron microscopic findings in naturally occurring virulent systemic feline calicivirus infection in cats, Vet. Pathol. (2004) 41:257-263.

[79] Poulet H., Brunet S., Soulier M., Leroy V., Goutebroze S., Chappuis G., Comparison between acute oral/respiratory and chronic stomatitis/gingivitis isolates of feline calicivirus: pathogenicity, antigenic profile and cross-neutralisation studies, Arch. Virol. (2000) 145:243-261.

[80] Poulet H., Brunet S., Leroy V., Chappuis G., Immunisation with a combination of two complementary feline calicivirus strains induces a broad cross-protection against heterologous challenges, Vet. Microbiol. (2005) 106:17-31.

[81] Povey C., Ingersoll J., Cross-protection among feline caliciviruses, Infect. Immun. (1975) 11:877-885.

[82] Povey R.C., Serological relationships among feline caliciviruses, Infect. Immun. (1974) 10:1307-1314.

[83] Povey R.C., Effect of orally administered ribavirin on experimental feline calicivirus infection in cats, Am. J. Vet. Res. (1978) 39:1337-1341.

[84] Radford A.D., Bennett M., McArdle F., Dawson S., Turner P.C., Glenn M.A., Gaskell R.M., The use of sequence analysis of a feline calicivirus (FCV) hypervariable region in the epidemiological investigation of FCV related disease and vaccine failures, Vaccine (1997) 15:1451-1458.

[85] Radford A.D., Turner P.C., Bennett M., McArdle F., Dawson S., Glenn M.A., Williams R.A., Gaskell R.M., Quasispecies evolution of a hypervariable region of the feline calicivirus capsid gene in cell culture and in persistently infected cats, J. Gen. Virol. (1998) 79:1-10.

[86] Radford A.D., Willoughby K., Dawson S., McCracken C., Gaskell R.M., The capsid gene of feline calicivirus contains linear B- cell epitopes in both variable and conserved regions, J. Virol. (1999) 73:8496-8502.

[87] Radford A.D., Dawson S., Wharmby C., Ryvar R., Gaskell R.M., Comparison of serological and sequence-based methods for typing feline calicivirus isolates from vaccine failures, Vet. Rec. (2000) 146:117123.

[88] Radford A.D., Sommerville L.M., Dawson S., Kerins A.M., Ryvar R., Gaskell R.M., Molecular analysis of isolates of feline calicivirus from a population of cats in a rescue shelter, Vet. Rec. (2001) 149:477-481.

[89] Radford A.D., Sommerville L., Ryvar R., Cox M.B., Johnson D.R., Dawson S., Gaskell R.M., Endemic infection of a cat colony with a feline calicivirus closely related to an isolate used in live attenuated vaccines, Vaccine (2001) 19:4358-4362.

[90] Radford A.D., Dawson S., Ryvar R., Coyne K., Johnson D.R., Cox M.B., Acke E.F., Addie D.D., Gaskell R.M., High genetic diversity of the immunodominant region of the feline calicivirus capsid gene in endemically infected cat colonies, Virus Genes (2003) 27:145-155.

[91] Reubel G.H., Hoffmann D.E., Pedersen N.C., Acute and chronic faucitis of domestic cats: a feline calicivirus induced disease, Vet. Clin. North Am. Small Anim. Pract. (1992) 22:1347-1360.

[92] Rice C.C., Kruger J.M., Venta P.J., Vilnis A., Maas K.A., Dulin J.A., Maes R.K., Genetic characterization of 2 novel feline caliciviruses isolated from cats with idiopathic lower urinary tract disease, J. Vet. Intern. Med. (2002) 16:293-302.

[93] Roerink F., Hashimoto M., Tohya Y., Mochizuki M., Genetic analysis of a canine calicivirus: evidence for a new clade of animal caliciviruses, Vet. Microbiol. (1999) 69:69-72.

[94] Sato Y., Ohe K., Murakami M., Fukuyama M., Furuhata K., Kishikawa S., Suzuki Y., Kiuchi A., Hara M., Ishikawa Y., Taneno A., Phylogenetic analysis of field isolates of feline calicivirus (FCV) in Japan by sequencing part of its capsid gene, Vet. Res. Commun. (2002) 26:205-219.

[95] Schorr-Evans E.M., Poland A., Pedersen N.C., An epizootic of highly virulent feline calicivirus disease in a hospital setting in New England, J. Feline Med. Surg. (2003) 5:217-226.

[96] Scott F.W., Virucidal disinfectants and feline viruses, Am. J. Vet. Res. (1980) 41:410-414. 
[97] Scott F.W., Geissinger C.M., Duration of immunity in cats vaccinated with an inactivated feline panleukopenia, herpesvirus and calicivirus vaccine, Feline Pract. (1997) 25:12-19.

[98] Scott F.W., Geissinger C.M., Long-term immunity in cats vaccinated with an inactivated trivalent vaccine, Am. J. Vet. Res. (1999) 60:652-658.

[99] Seal B.S., Ridpath J.F., Mengeling W.L., Analysis of feline calicivirus capsid protein genes: identification of variable antigenic determinant regions of the protein, J. Gen. Virol. (1993) 74:2519-2524.

[100] Sommerville L.M., Radford A.D., Glenn M., Dawson S., Gaskell C.J., Kelly D.F., Cripps P.J., Porter C.J., Gaskell R.M., DNA vaccination against feline calicivirus infection using a plasmid encoding the mature capsid protein, Vaccine (2002) 20:17871796.

[101] Sosnovtsev S., Green K.Y., RNA transcripts derived from a cloned full-length copy of feline calicivirus genome do not require $\mathrm{VpG}$ for infectivity, Virology (1995) 210:383-390.

[102] Sosnovtsev S.V., Sosnovtseva S.A., Green K.Y., Cleavage of the feline calicivirus capsid precursor is mediated by a virusencoded proteinase, J. Virol. (1998) 72:3051-3059.

[103] Sosnovtsev S.V., Green K.Y., Identification and genomic mapping of the ORF3 and VPg proteins in feline calicivirus virions, Virology (2000) 277:193-203.

[104] Sosnovtsev S.V., Prikhod'ko E.A., Belliot G., Cohen J.I., Green K.Y., Feline calicivirus replication induces apoptosis in cultured cells, Virus Res. (2003) 94:1-10.

[105] Sykes J.E., Studdert V.P., Browning G.F., Detection and strain differentiation of feline calicivirus in conjunctival swabs by RT-PCR of the hypervariable region of the capsid protein gene, Arch. Virol. (1998) 143:1321-1334.

[106] Taira O., Suzuki M., Takeuchi Y., Aramaki Y., Sakurai I., Watanabe T., Motokawa K., Arai S., Sato H., Maehara N., Expression of feline interferon-alpha subtypes in Escherichia coli, and their antiviral activity and animal species specificity, J. Vet. Med. Sci. (2005) 67:543-545.

[107] Tenorio A.P., Franti C.E., Madewell B.R., Pedersen N.C., Chronic oral infections of cats and their relationship to persistent oral carriage of feline calici-, immunodeficiency, or leukemia viruses, Vet. Immunol. Immunopathol. (1991) 29:1-14.
[108] TerWee T., Lauritzen A., Sabara M., Dreier K.J., Kokjohn K., Comparison of the primary signs induced by experimental exposure to either a pneumotrophic or a "limping" strain of feline calicivirus, Vet. Microbiol. (1997) 56:33-45.

[109] Thompson R.R., Wilcox G.E., Clark W.T., Jansen K.L., Association of calicivirus infection with chronic gingivitis and pharyngitis in cats, J. Small Anim. Pract. (1984) 25:207-210.

[110] Tohya Y., Yokoyama N., Maeda K., Kawaguchi Y., Mikami T., Mapping of antigenic sites involved in neutralization on the capsid protein of feline calicivirus, J. Gen. Virol. (1997) 78:303-305.

[111] Umehashi M., Imamura T., Akiyama S., Kimachi K., Tokiyoshi S., Mikami T., Postexposure treatment of cats with mousecat chimeric antibodies against feline herpesvirus type 1 and feline calicivirus, J. Vet. Med. Sci. (2002) 64:1017-21.

[112] Umehashi M., Imamura T., Akiyama S., Matsuda J., Tokiyoshi S., Tohya Y., Mikami T., Pre-exposure treatment of cats with antiFHV-1 and anti-FCV mouse-cat chimeric antibodies, J. Vet. Med. Sci. (2003) 65:563566.

[113] Wardley R.C., Gaskell R.M., Povey R.C., Feline respiratory viruses - their prevalence in clinically healthy cats, J. Small Anim. Pract. (1974) 15:579-586.

[114] Wardley R.C., Feline calicivirus carrier state: A study of the host/virus relationship, Arch. Virol. (1976) 52:243-249.

[115] Wardley R.C., Povey R.C., The clinical disease and patterns of excretion associated with three different strains of feline calicivirus, Res. Vet. Sci. (1977) 23:7-14.

[116] Waters L., Hopper C.D., Gruffydd-Jones T.J., Harbour D.A., Chronic gingivitis in a colony of cats infected with feline immunodeficiency virus and feline calicivirus, Vet. Rec. (1993) 132:340-342.

[117] Willcocks M.M., Carter M.J., Roberts L.O., Cleavage of eukaryotic initiation factor eIF4G and inhibition of host-cell protein synthesis during feline calicivirus infection, J. Gen. Virol. (2004) 85:1125-30.

[118] Yokoyama N., Fujita K., Damiani A., Sato E., Kurosawa K., Miyazawa T., Ishiguro S., Mochizuki M., Maeda K., Mikami T., Further development of a recombinant feline herpesvirus type 1 vector expressing feline calicivirus immunogenic antigen, $J$. Vet. Med. Sci. (1998) 60:717-723. 